\title{
Structural switching electrochemical DNA aptasensor for the rapid diagnosis of tuberculous meningitis
}

This article was published in the following Dove Medical Press journal: International Journal of Nanomedicine

\author{
Ritu Das,' Abhijeet \\ Dhiman, ${ }^{2,3}$ Subodh Kumar \\ Mishra, ${ }^{4}$ Sagarika Haldar, ${ }^{5,6}$ \\ Neera Sharma, ${ }^{7}$ Anjali \\ Bansal, ${ }^{7}$ Yusra Ahmad, ${ }^{3}$ Amit \\ Kumar, ${ }^{4}$ Jaya Sivaswami \\ Tyagi, ${ }^{2,5}$ Tarun Kumar \\ Sharma ${ }^{1,5}$
}

'AptaBharat Innovation Pvt. Ltd., Faridabad, India; ${ }^{2}$ Department of Biotechnology, All India Institute of Medical Sciences (AlIMS), New Delhi, India; ${ }^{3}$ Faculty of Pharmacy, Uttarakhand Technical University (UTU), Dehradun, India; ${ }^{4}$ Discipline of Biosciences and Biomedical Engineering, Indian Institute of Technology Indore, Simrol, India; ${ }^{5}$ Center for Biodesign and Diagnostics, Translational Health Science and Technology Institute, Faridabad, India; ${ }^{6}$ Department of Experimental Medicine and Biotechnology, Post Graduate Institute of Medical Education \& Research, Chandigarh, India; ${ }^{7} \mathrm{Dr}$ Ram Manohar Lohia Hospital, New Delhi, India

Correspondence: Jaya Sivaswami Tyagi Department of Biotechnology, All India Institute of Medical Sciences (AIIMS),

I 10029 New Delhi, India

$\mathrm{Tel}+9$ | 0112658849 |

Email jayatyagi.aiims@gmail.com

Tarun Kumar Sharma AptaBharat Innovation Pvt. Ltd., Translational Health Science and Technology Institute, NCR Biotech Cluster, 3rd Milestone, Faridabad I2 I00I, India

Tel +9l 01292876370

Email tarun@thsti.res.in
Background: Tuberculous meningitis (TBM) is the most devastating manifestation of extrapulmonary tuberculosis. About $33 \%$ of TBM patients die due to very late diagnosis of the disease. Conventional diagnostic methods based on signs and symptoms, cerebrospinal fluid (CSF) smear microscopy or liquid culture suffer from either poor sensitivity or long turnaround time (up to 8 weeks). Therefore, in order to manage the disease efficiently, there is an urgent and unmet need for a rapid and reliable diagnostic test.

Methods: In the current study, to address the diagnostic challenge of TBM, a highly rapid and sensitive structural switching electrochemical aptasensor was developed by combining the electrochemical property of methylene blue (MB) with the molecular recognition ability of a ssDNA aptamer. To demonstrate the clinical diagnostic utility of the developed aptasensor, a blinded study was performed on 81 archived CSF specimens using differential pulse voltammetry.

Results: The electrochemical aptasensor developed in the current study can detect as low as $10 \mathrm{pg}$ HspX in CSF background and yields a highly discriminatory response $(P<0.0001)$ for TBM and not-TBM categories with $\sim 95 \%$ sensitivity and $\sim 97.5 \%$ specificity and has the ability to deliver sample-to-answer in $\leq 30$ minutes.

Conclusion: In summary, we demonstrate a new aptamer-based electrochemical biosensing strategy by exploiting the target-induced structural switching of H63 SL-2 M6 aptamer and electroactivity of aptamer-tagged MB for the detection of HspX in CSF samples for the diagnosis of TBM. Further, the clinical utility of this sensor could be extended for the diagnosis of other forms of tuberculosis in the near future.

Keywords: aptamers, Mycobacterium tuberculosis, TB meningitis, clinical diagnostic, electrochemical sensing

\section{Introduction}

Tuberculosis (TB) is a highly infectious disease that causes significant morbidity and mortality worldwide with an estimated 10.4 million new cases and over 1.7 million deaths in 2016. TB continues to pose an enormous threat to public health management systems across the globe, especially in the developing world. ${ }^{1}$

Tuberculous meningitis (TBM) is the most lethal manifestation of extrapulmonary tuberculosis that causes irreversible damage to the central nervous system (CNS) and has an estimated mortality of 1.5 per 100,000 population in India. ${ }^{2,3}$ Children and immunocompromised adults are at higher risk of acquiring TBM infection. TBM leads to delayed CNS neurological complications including, but not limited to, cerebral abscess, neurological sequelae, hydrocephalus, etc, and ultimately to death. ${ }^{4}$ About $33 \%$ of TBM patients die due to the delayed diagnosis of the disease. Therefore, in 
order to manage the disease efficiently, there is an urgent and unmet need for a rapid and reliable diagnostic test.

Conventional diagnostic methods for TBM mainly rely on presentation of signs and symptoms that are often nonspecific and can resemble other meningoencephalitis, which could misguide the clinician. Other conventional approaches for TBM diagnosis include microbiological analysis of cerebrospinal fluid (CSF). ${ }^{5}$ However, microscopy exhibits poor sensitivity due to the paucibacillary nature of the disease, while CSF culture has a long turnaround time (up to 8 weeks). ${ }^{6}$ Furthermore, collection of an adequate volume of CSF sample from pediatric patients for culture is a major challenge for clinicians. ${ }^{3,7}$ A test that has gained some popularity in recent years for diagnosing TBM is Gene Xpert MTB/RIF (Xpert). Xpert is a rapid and automated PCR-based molecular test with a minimal requirement of trained workforce. However, only 55\% sensitivity with $84 \%$ negative predictive value (NPV) was obtained according to a recently performed meta-analysis of Xpert for TBM when a clinical gold standard was used. This NPV suggests that 1 in 6 TBM patients is being missed in high prevalence TBM settings when tested by Xpert. ${ }^{8-11}$ A newer advanced version ie, Xpert Ultra has shown a higher sensitivity but its applicability has been demonstrated only in HIV patients. ${ }^{12}$ Therefore, Xpert as a sole diagnostic test has not been recommended considering the current evidence, ${ }^{8}$ as delay in the treatment of TBM can lead to fatal consequences. Moreover, the high cost of the test and dependence on proprietary equipment and reagents limits the application of Xpert in resource-limited settings. ${ }^{3,13-15}$ Therefore, to address the aforementioned challenges, there is an urgent need of a facile, cost-effective, rapid and sensitive test for TBM diagnosis.

HspX is a Mycobacterium tuberculosis antigen (also known as $\alpha$-crystallin) which is abundantly expressed under hypoxia $^{3,14}$ and in both active and latent forms of TB. ${ }^{3,14,16}$ The utility of this antigen in the antibody-based diagnosis of pulmonary TB has already been established. ${ }^{17-19}$ In another study, the detection of HspX messenger RNA was used to detect viable $M$. tuberculosis directly in sputum specimens from clinically suspected TB patients. ${ }^{20}$ Notably, HspX antigen was considered as a reliable biomarker for TBM diagnosis in a polyclonal antibody-based ELISA using CSF. ${ }^{3}$ While this ELISA evinced a high diagnostic accuracy, a polyclonal antibody-based test has inherent problems that include batch-to-batch variation in antibody titer, limited stability and requirement of a cold chain. These limitations pose a significant challenge during translation and upscaling of laboratory findings into a point-of-care (POC) test for TBM. In recent years, functional nucleic acids known as aptamers (DNA or RNA) have demonstrated the ability to potentially overcome the limitations associated with antibodies. ${ }^{21-26}$ Owing to these attributes, aptamers are regarded as a strong chemical rival of antibodies, with an ability to replace antibodies in all possible diagnostic formats. ${ }^{22,27}$ Furthermore, in the last few years, aptamers have demonstrated their utility in the detection of diverse analytes ranging from small molecules to antigens to enzymes to hormones and even whole bacterial or cancer cells. ${ }^{28-30}$ The unique ability of aptamers to adapt on various diagnostic platforms without compromising their affinity and specificity make them diagnostic reagents of choice.

An ultrasensitive diagnostic platform is highly desirable to detect trace amounts of biomarker present in clinical specimens for the accurate diagnosis of disease. ${ }^{31-33}$ In recent years, the electrochemical detection platform has generated considerable interest owing to its ease of operation, high sensitivity (up to femtomole), rapid detection, negligible or no requirement of sample processing and the ability to provide both qualitative and quantitative output. ${ }^{32,34}$ In particular, methylene blue (MB) is useful as a redox probe in aptamer-based electrochemical sensors owing to its remarkable ability to participate in electron transfer, ease in modification of the aptamer by MB, high electroactivity and stability. ${ }^{35}$

In the current study, we utilize the electron transfer property of $\mathrm{MB}$ in combination with target-induced structural switching ability of an in-house generated HspX specific DNA aptamer (H63 SL2-M6) ${ }^{36}$ for the detection of HspX in CSF samples for the diagnosis of TBM. The secondary structure of this aptamer is shown in Figure S1.

\section{Methods}

\section{Reagent and materials}

Gold (III) chloride $\left(\mathrm{HAuCl}_{4} \cdot 3 \mathrm{H}_{2} \mathrm{O}\right), 2$-Mercaptoethanol $(\beta-\mathrm{ME})$, Tris- $\mathrm{HCl}, \mathrm{KCl}, \mathrm{MgCl}_{2}, \mathrm{NaCl}$ and other reagents used in this study were procured from Sigma-Aldrich (SigmaAldrich Co., St Louis, MO, USA). Potassium ferrocyanide $\left(\mathrm{K}_{4} \mathrm{Fe}(\mathrm{CN})_{6} \cdot 3 \mathrm{H}_{2} \mathrm{O}\right)$ and potassium ferricyanide $\mathrm{K}_{3} \mathrm{Fe}(\mathrm{CN})_{6}$ were obtained from HiMedia laboratories (Mumbai, India). HspX specific aptamer (H63 SL2 M6) was custom synthesized from LGC Biosearch (Petaluma, CA, USA) with 5' $\mathrm{MB}$ and 3' C6 thiol modification. A screen-printed electrode system (SPE) comprising carbon as working electrode (WE; $\Phi=3 \mathrm{~mm}$ ), platinum as auxiliary electrode and $\mathrm{Ag}$ / $\mathrm{AgCl}$ as a reference electrode was procured from ItalSens (PalmSens BV, Houten, the Netherlands) and was used to build an aptamer-based electrochemical sensor. Hexa-His 
tagged HspX protein was expressed and purified in recombinant from Escherichia coli DH5 $\alpha$ using standard Ni-NTA chromatography.

\section{Instrumentation}

Electrochemical measurements were carried out for characterizing the fabrication of the sensing interface using an EmstatBlue (PalmSens) equipped with PSTrace software. The aforementioned SPE system was used for electrochemical sensing assays after electrodeposition of gold nanoparticles (GNPs) cyclic voltammetry (CV) were performed in a solution of $5 \mathrm{~mm}\left[\mathrm{Fe}(\mathrm{CN})_{6}{ }^{3-/ 4-}\right]$ and differential pulse voltammetry (DPV) readings were recorded in binding buffer (10 mM Tris- $\mathrm{HCl}$ supplemented with $50 \mathrm{mM} \mathrm{KCl,} 25 \mathrm{mM}$ $\mathrm{NaCl}$ and $10 \mathrm{mM} \mathrm{MgCl}_{2}$ ).

\section{Fabrication and characterization of sensing platform}

The electrodeposition of GNPs was carried out for providing a platform for immobilization of thiol-labelled aptamer on carbon SPE electrode using an aqueous solution of 1.0 $\mathrm{mM} \mathrm{HAuCl}_{4}$ in the presence of $0.5 \mathrm{M} \mathrm{H}_{2} \mathrm{SO}_{4}$ at a constant potential of $-0.2 \mathrm{~V}$ for 200 seconds. After washing and drying of GNP-deposited SPE, the electrode was characterized using a scanning electron microscope (SEM) and CV. The morphology of the GNP-modified electrodes were observed using SEM (Zeiss evo 18; Carl Zeiss Microscopy, LLC, Thornwood, NY, USA). Further, the screen-printed electrode was characterized before and after deposition of GNPs by CV for electrochemical characterization in $5 \mathrm{mM}\left[\mathrm{Fe}(\mathrm{CN})_{6}^{3-/ 4-}\right]$. A potential ranging from $-0.4 \mathrm{~V}$ to $0.8 \mathrm{~V}$ was applied to record the cyclic voltammogram. Following electrochemical deposition of GNPs, $5.0 \mu \mathrm{L}$ of thiol-activated aptamer $(10 \mu \mathrm{M})$ in binding buffer was placed on the modified SPE and was incubated at $30^{\circ} \mathrm{C}$ overnight. Then the aptamermodified electrode was thoroughly rinsed with binding buffer followed by distilled water to remove the weakly adsorbed aptamer and dried. The aptamer-modified electrode was further exposed to $10 \mathrm{mM}$ 2-mercaptoethanol ( $\beta$-ME) for 20 minutes to block the uncovered electrode surface. Finally, the modified electrode was rinsed thoroughly with binding buffer followed by double distilled water.

\section{Electrochemical detection of HspX}

The prepared SPE-based sensing interface was incubated with $100 \mathrm{ng} \mathrm{HspX}$ protein for 15 minutes at room temperature. The sensing interface was incubated with nine differentantigens (HspX, ESAT-6, CFP-10, MPT-64, MPT-51, Ag85complex, GroES, CFP and LAM of M. tuberculosis) and incubated for 15 minutes for determination of selectivity of the sensor. After incubation, the electrodes were washed with binding buffer carefully to remove the unbound protein. In order to detect bound protein, DPV was performed.

\section{Limit of detection}

In order to determine the low-end detection limit, first a range (0.01-500 ng) of HspX was spiked in binding buffer and DPV was performed. Further, to study the effect of sample matrix on aptamer-HspX interaction, pooled CSF samples obtained from noninfectious neurological disorder subjects (NIND) was diluted (1:10 in binding buffer) and then it was spiked with HspX (0.01-500 ng). Following this, DPV was performed to determine the lowest possible amount of HspX that can be detected in the CSF.

\section{Application of the aptasensor in clinical assay}

To detect HspX level in clinical samples, archived CSF samples were used as described in classification of CSF samples section (Supplementary materials). These CSF samples were diluted to $1: 10$ in binding buffer $(10 \mathrm{mM}$ Tris $\mathrm{pH} 7.5$, $25 \mathrm{mM} \mathrm{NaCl}, 10 \mathrm{mM} \mathrm{MgCl}_{2}, 50 \mathrm{mM} \mathrm{KCl}$ ) before performing the experiment (DPV).

\section{Ethical statement}

Ethical clearance for evaluating the antigen-based test to detect TBM was taken from the Institutional Ethics Committee (AIIMS and RMLH). Since the current study was conducted on pediatric CSF samples, all samples were collected after obtaining a written informed consent from parents of the subjects. Patient information was collected in a confidential manner as per ethical principles of the Declaration of Helsinki of 1964 revised by the 59th WMA General Assembly, Seoul, in October 2008.

\section{Results and discussion Principle of structural switching aptasensor}

The schematic in Figure 1 illustrates the principle of the aptamer-based electrochemical biosensor. A dual-labelled aptamer (H63 SL2-M6 with 5' MB and 3' thiol label having C6 spacer) is immobilized on to the disposable SPE having gold-coated carbon WE. In the absence of HspX protein, the MB-labelled end of the aptamer is in the close proximity of the WE, resulting in high electron transfer. However, upon encounter with HspX in the CSF sample, a target-induced structural change in the aptamer occurs, which increases the distance between $\mathrm{MB}$ and $\mathrm{WE}$, causing a rapid decrease 

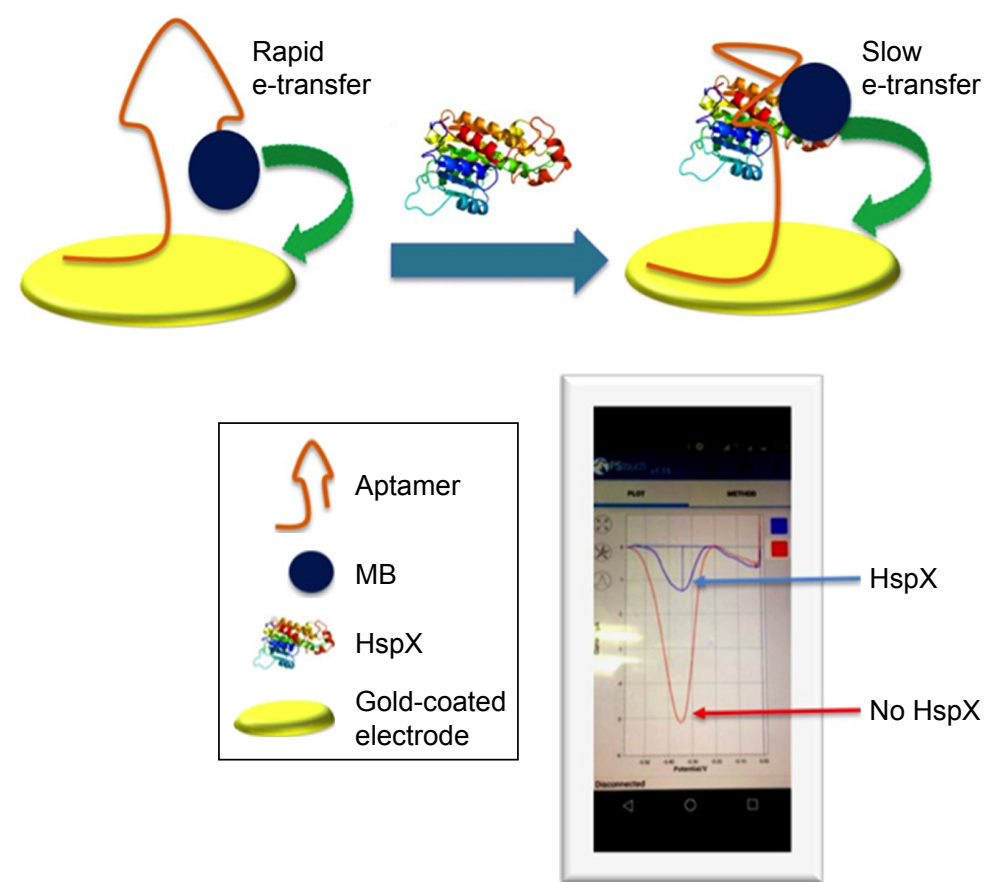

Figure I Schematic representation of the aptamer-based electrochemical sensor for the detection of HspX antigen in CSF.

Notes: The aptamer is thiol-labelled at the $3^{\prime}$ end that allows self-assembly of aptamer on a gold-coated electrode. The redox label (MB) is attached at the $5^{\prime}$ of the aptamer and it is in close proximity to the electrode surface, thereby allowing rapid electron transfer. The presence of HspX induces a structural change in the aptamer which increases the distance between $\mathrm{MB}$ and the electrode, and electron transfer is thus reduced. The differences in faradaic current before and after addition of HspX were quantified using DPV.

Abbreviations: MB, methylene blue; CSF, cerebrospinal fluid; DPV, differential pulse voltammetry.

in electron transfer, thereby reducing the current. Thus, in presence of the cognate target HspX, a turn-off response is achieved, which forms the basis of electrochemical sensing of HspX.

\section{Circular dichroism (CD) study}

At first, the HspX-induced structural change in the aptamer was monitored using circular dichroism (CD). A negative peak at $246 \mathrm{~nm}$ and two positive peaks at 220 and $272 \mathrm{~nm}$ were recorded (Figure 2), a typical signature of stem loop/ hairpin type B-DNA structure. ${ }^{37}$ In the presence of HspX, a structural change is indicated by the change in molecular ellipticity suggesting that the aptamer can work as a molecular switch for the detection of HspX.

\section{Electrode characterization}

The SEM characterization of the bare SPE shows a rough surface, while electrodeposition of gold chloride leads to coating of spherical GNPs on the electrode surface (Figure 3). CV further confirmed the deposition of GNPs on the electrode; an increase in current was recorded on the GNP-coated electrode in comparison to the bare electrode due to the increased conductivity of gold (Figure 4). These results are in concordance with the previously published reports. ${ }^{38}$

\section{Evaluation of aptamer specificity}

Thereafter, the dual-labelled (MB- and thiol-labelled) H63 SL2-M6 aptamer was immobilized on the gold-coated electrode and used to evaluate the specificity of the sensor. The sensor response in the presence of various M. tuberculosis antigens (HspX, ESAT-6, CFP-10, MPT-64, MPT-51, Ag85complex, GroES, CFP and LAM) was compared and

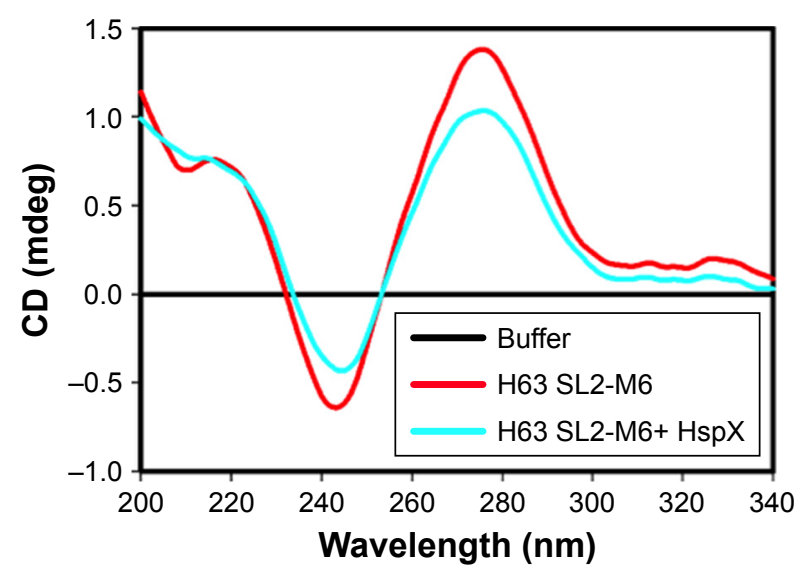

Figure $2 \mathrm{CD}$ spectrum for free aptamer $(10 \mu \mathrm{M})$ and aptamer in the presence of HspX $(20 \mu M)$.

Note: In the presence of HspX, the aptamer undergoes target-induced structural change as evident by change in ellipticity.

Abbreviation: $C D$, circular dichroism. 


\section{Bare electrode}

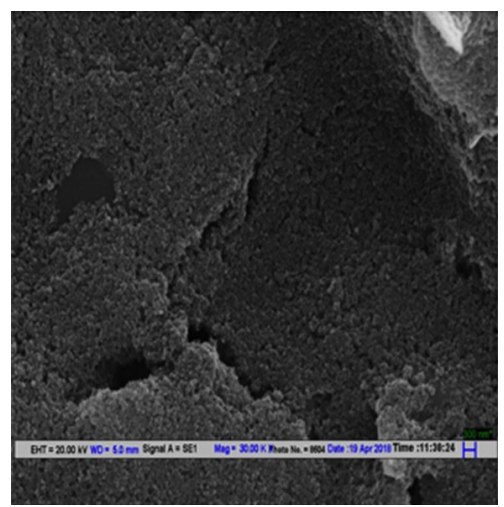

Gold-coated electrode at $3000 \mathrm{~K}$

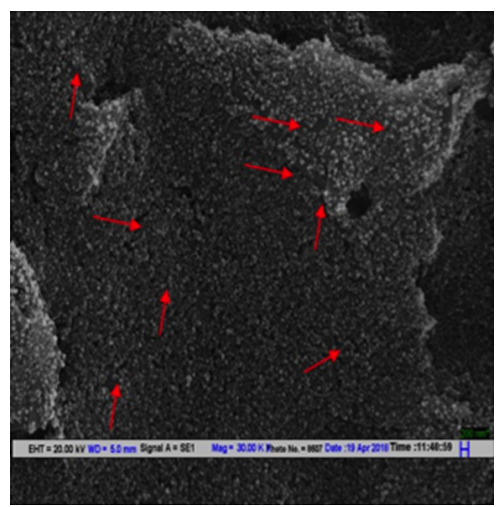

Gold-coated electrode at $5000 \mathrm{~K}$

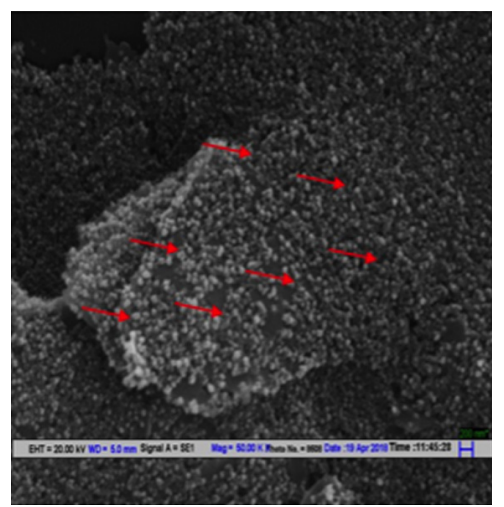

Figure 3 Scanning electron micrograph of SPE, before and after electrodeposition of gold (at 3000K and 5000K magnification).

Notes: After electrodeposition, spherical GNPs are visible on the electrode surface while bare electrode presents a rough surface. Red arrows point to representative GNPs deposited on the SPE surface.

Abbreviations: SPE, screen-printed electrodes; GNPs, gold nanoparticles.

is represented as a three-color gradient heat map (Figure 5). The sensor response is highly selective for HspX and a sharp decrease in current was observed only in presence of HspX. Such a change was not observed in presence of the other antigens that are expressed during TB infection, such as MPT-51, GlcB, CFP-10 and ESAT-6, or secretory proteins such as $\mathrm{Ag} 85 \mathrm{C}$ and culture filtrate proteins, or GroES heat shock protein or LAM, a major component of bacterial cell wall.

\section{Determining limit of HspX detection}

The sensitivity of HspX detection using the electrochemical sensing approach was determined next. The DPV of M6

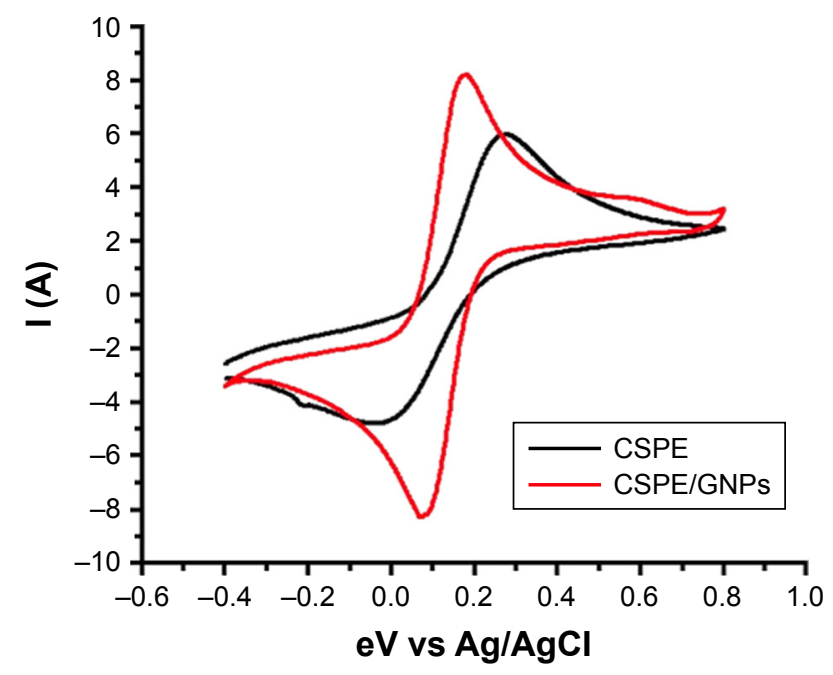

Figure 4 Cyclic voltammogram of CSPE before (black) and after (red) electrodeposition of GNPs.

Note: Electrodeposition of gold makes the electrode surface more conductive, resulting in an increase in current response.

Abbreviations: CSPE, carbon-screen-printed electrode; $\mathrm{Ag} / \mathrm{AGCl}$, silver/silver chloride; GNPs, gold nanoparticles. aptamer-modified electrode was recorded in the presence and absence of HspX (500 ng) (Figure S2). The aptamerbased electrochemical sensor was highly sensitive and able to detect as low as $10 \mathrm{pg}$ of HspX (Figure S3). Following this, a range of HspX protein (0.01-500 ng) spiked into CSF (obtained from not-TBM subjects belonging to noninfectious neurological disorders category, described under "Classification of CSF samples" in Supplementary materials) was evaluated to assess the effect of clinical sample matrix on sensor sensitivity. Notably, the aptasensor exhibited a similar low-end detection limit of $10 \mathrm{pg} \mathrm{HspX}$ in CSF background (Figure S4). However, the signal strength was marginally reduced in the CSF background, suggesting the possibility of mild quenching of the signal by the sample matrix.

\begin{tabular}{|l|c|}
\multicolumn{1}{c}{} & \multicolumn{1}{c}{$\Delta 21$} \\
\hline HspX & 321 \\
\hline CFP-10 & 0 \\
\hline ESAT-6 & 0 \\
\hline Ag85 & 0 \\
\hline GroES & 0 \\
\hline MPT-64 & 0 \\
\hline MPT-51 & 0 \\
\hline CFP & 0 \\
\hline LAM & 0 \\
\hline
\end{tabular}

Figure $5 \mathrm{~A}$ three-color gradient heat-map representation of sensor response. Notes: Red color represents highest sensor response while blue indicates the weakest sensor response. The current difference values are also displayed for each antigen. The current difference values obtained in negatives are considered as ' 0 '. 


\section{Evaluation of aptasensor in clinical specimens}

Finally, to demonstrate the clinical diagnostic utility of the developed aptasensor, its performance was assessed in a blinded manner in 81 archived CSF specimens from pediatric subjects (Supporting information Table S1) belonging to TBM and not-TBM disease control categories, as described in the Supplementary materials section under "Classification of CSF samples". An ethical permission was obtained from the institutional ethics committee for the use of CSF samples in the current study. A receiver operating characteristic (ROC) curve was constructed using $\Delta \mathrm{I} / \mathrm{mA}$ values (current difference before and after adding the sample) obtained with CSF from definite (true positive) and nontuberculous infectious meningitis (NTIM) group (true negative) samples. The area under the curve (AUC) was 1.0 (Figure S5) and established that the assay is highly robust. The NTIM category comprised of 16 cases of pyogenic bacterial meningitis that included 14 cases that were diagnosed on the basis of response to appropriate antibiotics, clinical presentation along with symptoms and culture confirmed cases of $E$. coli $(\mathrm{N}=1)$, and Acinetobacter $\mathrm{sp}$. $(\mathrm{N}=1)$. Based on the cut-off derived from the ROC curve, the performance of the electrochemical sensor was evaluated in all 81 samples (Figure 6). The test yielded a highly discriminatory response $(P<0.0001)$ for TBM and not-TBM category with $\sim 95 \%$ sensitivity and $\sim 97.5 \%$ specificity. A recent assessment of Xpert on CSF samples showed its performance to be modest with a sensitivity of $\sim 55 \% .{ }^{39}$ While a newer version of Xpert MTB/RIF ie, Xpert Ultra exhibited 70\% sensitivity for probable or definite TBM subjects having

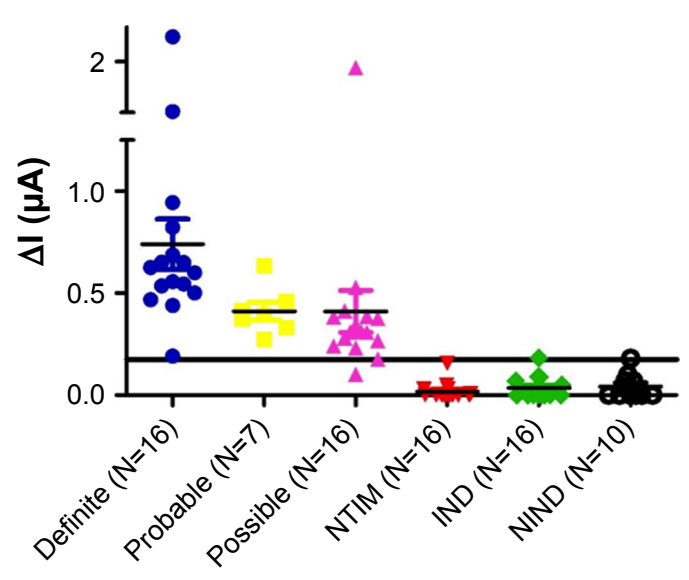

Figure 6 Performance of H63 SL-2 M6 aptamer-based electrochemical sensor on archived CSF samples obtained from TBM patients ( $N=39$; Definite $N=16$, Probable $\mathrm{N}=7$, Possible $\mathrm{N}=16$ ) and Not-TBM patients $(\mathrm{N}=42$; NTIM $N=16$, IND $N=16$ and NIND N=10).

Abbreviations: CSF, cerebrospinal fluid; IND, infectious neurological disorders; NIND, nonIND; TBM, tuberculous meningitis.
HIV compared to a uniform case definition. ${ }^{12}$ In comparison, the aptamer-based electrochemical sensor developed in this study was significantly superior with $\sim 95 \%$ sensitivity. This high sensitivity of our diagnostic test is likely due to the high affinity of the aptamer for the target, electrochemical sensing platform and high HspX load present is CSF of TMB subjects. A recent study from our group suggests that HspX is present in a range of $3 \times 10^{6} \mathrm{fg}(3 \mathrm{ng})$ to $2.8 \times 10^{8} \mathrm{fg}$ (280 ng) in $5 \mu \mathrm{L} \mathrm{CSF} \mathrm{sample.}{ }^{3}$ A comparison of the performance of the aptamer-based test reported here with previously reported TBM diagnostic tests is shown in Table 1. Moreover, owing to its simple format, the developed electrochemical sensor meets the requirements of a rapid portable bedside diagnostic test for TBM. Importantly, the electrochemical sensor designed in the current study requires a very small volume of CSF $(\sim 4 \mu \mathrm{L})$ and addresses the challenge of collecting a large volume of CSF for various diagnostic investigations. In comparison to the existing methods, the test developed in the current study sampleto-answer time of $\leq 30$ minutes with high selectivity and sensitivity. Further, the developed assay can be operated through a mobile phone that makes it a truly POC assay and can also help in rapid dissemination of results and clinical decision-making.

\section{Conclusion}

In summary, we demonstrate a new aptamer-based electrochemical biosensing strategy by exploiting the target-induced structural switching of H63 SL-2 M6 aptamer and electroactivity of aptamer-tagged MB for the detection of HspX in CSF samples for the diagnosis of TBM. This sensor exhibits high sensitivity and specificity ( $\sim 95 \%$ and $\sim 97.5 \%$, respectively) and sample-to-answer time of $\leq 30$ minutes. One limitation of this study was that the ROC derived cut-off was based on the studied sample size $(\mathrm{N}=81)$. However, in order to derive a more robust cut-off we propose to conduct an independent validation of the developed assay on blinded sample sets from geographically distinct populations of TBM patients in the near future. The findings of this proof of concept study clearly indicate the translational potential of the developed electrochemical aptasensor for the detection of TBM, and in future, its application may also be extended for the diagnosis of other forms of tuberculosis.

\section{Acknowledgments}

This work was supported through a Biotechnology Ignition Grant (BIRAC/IKP0366/BIG-08/16) from the Biotechnology Research Assistance Council (BIRAC) to AptaBharat Innovation Pvt. Ltd. and funding support through the National 
Table I Performance of developed tests in diagnosis of TBM

\begin{tabular}{|c|c|c|c|}
\hline Assays for TBM diagnosis & Sensitivity (\%) & Specificity (\%) & Comments \\
\hline TB culture ${ }^{40}$ & 27 & & Poor sensitivity \\
\hline Smear microscopy ${ }^{41}$ & $10-20$ & & Poor sensitivity \\
\hline \multicolumn{4}{|l|}{ NAATs } \\
\hline Xpert MTB/RIF assay ${ }^{39}$ & 55.1 & 94.8 & Moderate sensitivity, but an excellent rule-in test \\
\hline $\begin{array}{l}\text { Nucleic acid commercial } \\
\text { amplification tests }{ }^{42} \\
\text { meta-analysis }\end{array}$ & 56 & 98 & \multirow[t]{2}{*}{$\begin{array}{l}\text { Suboptimal sensitivity, time-consuming, requires DNA } \\
\text { isolation and amplification steps }\end{array}$} \\
\hline $\begin{array}{l}\text { Commercial NAATs }{ }^{43} \text { meta- } \\
\text { analysis }\end{array}$ & 64 & 98 & \\
\hline In-house qPCR ${ }^{44}$ & $\begin{array}{l}87.6 \text { (filtrate) } \\
53.1 \text { (sediment) }\end{array}$ & 92 & \multirow{2}{*}{$\begin{array}{l}\text { Time-consuming, requires DNA isolation and amplification } \\
\text { steps } \\
\text { Time-consuming, requires DNA isolation and amplification } \\
\text { steps }\end{array}$} \\
\hline In-house qPCR ${ }^{3}$ & $\begin{array}{l}100 \\
\text { (definite TBM) } \\
98 \text { (probable and } \\
\text { possible TBM) }\end{array}$ & $\begin{array}{l}\text { 96-97 } \\
\text { (definite TBM) } \\
98 \text { (probable and } \\
\text { possible TBM) }\end{array}$ & \\
\hline \multicolumn{4}{|l|}{ Antigen detection tests } \\
\hline This study & 95 & 97.5 & $\begin{array}{l}\text { Excellent diagnostic accuracy, laboratory-based format } \\
\text { adaptable to POC, only } 30 \text { minutes from sample to answer }\end{array}$ \\
\hline $\begin{array}{l}\text { Aptamer-based ALISA } \\
\text { HspX }{ }^{36}\end{array}$ & 100 & 91 & Excellent diagnostic accuracy, laboratory-based format \\
\hline $\begin{array}{l}\text { Antibody-based ELISA } \\
\text { GlcB, HspX and PstSI }\end{array}$ & $\begin{array}{l}100 \\
\text { (definite TBM) }\end{array}$ & $\begin{array}{l}\text { 96-97 } \\
\text { (definite TBM) }\end{array}$ & $\begin{array}{l}\text { Excellent diagnostic accuracy, laboratory-based format, } \\
\text { time-consuming, uses antibody which might have batch to } \\
\text { batch variation }\end{array}$ \\
\hline GlcB, HspX and MPT5I & $\begin{array}{l}\text { 92-95 } \\
\text { (probable and possible } \\
\text { TBM) }\end{array}$ & $\begin{array}{l}93-96 \\
\text { (probable and possible } \\
\text { TBM) }\end{array}$ & \\
\hline $\begin{array}{l}\text { Antibody-based ELISA } \\
\text { LAM, Ag85 complex, } 65 \mathrm{kDa} \\
\text { and ESAT- } 6^{45}\end{array}$ & 87 & 84 & $\begin{array}{l}\text { Laboratory-based format, time-consuming, uses antibody } \\
\text { which might have batch to batch variation }\end{array}$ \\
\hline
\end{tabular}

Abbreviations: TBM, tuberculosis meningitis; TB, tuberculosis; NAATs, nucleic acid amplification tests; POC, point-of-care; ALISA, aptamer-linked immobilized sorbent assay.

Biodesign Alliance Project from the Department of Biotechnology, All India Institute of Medical Sciences, which the Government of India awarded to JST (BT/PR14638/ MED/12/483/2010). AD acknowledges the Indian Council of Medical Research (ICMR) for providing Senior Research Fellowship. The excellent technical assistance of Sanjay Kumar and Jitendra Kumar is duly acknowledged. Sarin Prakash and Sunil Kumar are sincerely thanked for their active involvement in data management and sample collection.

\section{Disclosure}

The aptamer used in this study is a proprietary reagent and is deposited in the Indian Patent Office as part of Indian Patent application number 201611001550; the patent was transferred to a Translational Health Science and Technology Institute (THSTI) spinoff, AptaBharat Innovation Pvt. Ltd. (ABIPL), for further development of aptamer-based diagnostics.
RD is employed by AptaBharat Innovation Pvt. Ltd (ABIPL) and TKS is employed by THSTI. TKS is also the founder of THSTI spinoff, ABIPL and owns $87 \%$ stakes in ABIPL. The authors report no other conflicts of interest in this work.

\section{References}

1. World Health Organization [homepage on the Internet]. Global tuberculosis report 2017. Available from: http://www.who.int/tb/publications/ global_report/en/. Accessed August 30, 2018.

2. Chakraborty AK. Estimating mortality from tuberculous meningitis in a community: use of available epidemiological parameters in the Indian context. Ind J Tub. 2000;47:9-14.

3. Haldar S, Sankhyan N, Sharma N, et al. Detection of Mycobacterium tuberculosis GlcB or HspX antigens or devR DNA impacts the rapid diagnosis of tuberculous meningitis in children. PLoS One. 2012;7(9): e44630.

4. Chin JH. Tuberculous meningitis: diagnostic and therapeutic challenges. Neurol Clin Pract. 2014;4(3):199-205.

5. Verdon R, Chevret S, Laissy JP, Wolff M. Tuberculous meningitis in adults: review of 48 cases. Clin Infect Dis. 1996;22(6):982-988.

6. Andersen P, Munk ME, Pollock JM, Doherty TM. Specific immune-based diagnosis of tuberculosis. The Lancet. 2000;356(9235):1099-1104. 
7. Marais S, Thwaites G, Schoeman JF, et al. Tuberculous meningitis: a uniform case definition for use in clinical research. Lancet Infect Dis. 2010;10(11):803-812.

8. Bahr NC, Marais S, Caws M, et al. GeneXpert MTB/RIF to diagnose tuberculous meningitis: perhaps the first test but not the last. Clin Infect Dis. 2016;62(9):1133-1135.

9. Sobotka O, Endlicher R, Drahota Z, et al. Impaired mitochondrial functions contribute to 3-bromopyruvate toxicity in primary rat and mouse hepatocytes. J Bioenerg Biomembr. 2016;48(4):363-373.

10. Patel VB, Theron G, Lenders L, et al. Diagnostic accuracy of quantitative PCR (Xpert MTB/RIF) for tuberculous meningitis in a high burden setting: a prospective study. PLoS Med. 2013;10(10):e1001536.

11. Nhu NT, Heemskerk D, Thu DDA, et al. Evaluation of GeneXpert MTB/RIF for diagnosis of tuberculous meningitis. J Clin Microbiol. 2014;52(1):226-233.

12. Bahr NC, Nuwagira E, Evans EE, et al. Diagnostic accuracy of Xpert MTB/RIF ultra for tuberculous meningitis in HIV-infected adults: a prospective cohort study. Lancet Infect Dis. 2018;18(1):68-75.

13. Kashyap RS, Kainthla RP, Satpute RM, et al. Demonstration of IgG antibodies to $30 \mathrm{kD}$ protein antigen in CSF for diagnosis of tuberculous meningitis by antibody-capturing ELISA. Neurol India. 2004;52(3): 359-362.

14. Chandramuki A, Bothamley GH, Brennan PJ, Ivanyi J. Levels of antibody to defined antigens of Mycobacterium tuberculosis in tuberculous meningitis. J Clin Microbiol. 1989;27(5):821-825.

15. Pai NP, Vadnais C, Denkinger C, Engel N, Pai M. Point-of-care testing for infectious diseases: diversity, complexity, and barriers in low- and middle-income countries. PLoS Med. 2012;9(9):e1001306.

16. Castro-Garza J, García-Jacobo P, Rivera-Morales LG, et al. Detection of anti-HspX antibodies and HspX protein in patient sera for the identification of recent latent infection by Mycobacterium tuberculosis. PLoS One. 2017;12(8):e0181714.

17. Laal S, Samanich KM, Sonnenberg MG, Zolla-Pazner S, Phadtare JM, Belisle JT. Human humoral responses to antigens of Mycobacterium tuberculosis: immunodominance of high-molecular-mass antigens. Clin Diagn Lab Immunol. 1997;4(1):49-56.

18. Singh KK, Dong Y, Belisle JT, Harder J, Arora VK, Laal S. Antigens of Mycobacterium tuberculosis recognized by antibodies during incipient, subclinical tuberculosis. Clin Diagn Lab Immunol. 2005;12(2):354-358.

19. Mori T, Sakatani M, Yamagishi F, et al. Specific detection of tuberculosis infection: an interferon-gamma-based assay using new antigens. Am J Respir Crit Care Med. 2004;170(1):59-64.

20. Lakshmipathy D, Kulandai LT, Ramasubban G, Hajib Narahari Rao M, Rathinam S, Narasimhan M. Nested reverse transcriptase-polymerase chain reactions targeting the messenger RNA of ICL2, hspx, and rRNAP1 genes to detect viable Mycobacterium tuberculosis directly from clinical specimens. Int J Mycobacteriol. 2015;4(4):312-317.

21. You M, Chen Y, Peng L, et al. Engineering DNA aptamers for novel analytical and biomedical applications. Chem Sci. 2011;2(6):1003.

22. Dhiman A, Kalra P, Bansal V, Bruno JG, Sharma TK. Aptamer-based point-of-care diagnostic platforms. Sensors Actuators B Chem. 2017; 246:535-553.

23. Seo HB, Gu MB. Aptamer-based sandwich-type biosensors. J Biol Eng. 2017;11:11.

24. Kumari P, Lavania S, Tyagi S, et al. A novel aptamer-based test for the rapid and accurate diagnosis of pleural tuberculosis. Anal Biochem. 2019;564-565:80-87.

25. Lavania S, Das R, Dhiman A, et al. Aptamer-based TB antigen tests for the rapid diagnosis of pulmonary tuberculosis: potential utility in screening for tuberculosis. ACS Infect Dis. 2018;4(12):1718-1726.
26. Kaur H, Bhagwat SR, Sharma TK, Kumar A. Analytical techniques for characterization of biological molecules - proteins and aptamers/ oligonucleotides. Bioanalysis. 2019;11(2):103-117.

27. Sharma TK, Bruno JG, Dhiman A. ABCs of DNA aptamer and related assay development. Biotechnol Adv. 2017;35(2):275-301.

28. Chen L, Rashid F, Shah A, et al. The isolation of an RNA aptamer targeting to p53 protein with single amino acid mutation. Proc Natl Acad Sci U S A. 2015;112(32):10002-10007.

29. Lee JF, Stovall GM, Ellington AD. Aptamer therapeutics advance. Curr Opin Chem Biol. 2006;10(3):282-289.

30. Kaur H, Bruno JG, Kumar A, Sharma TK. Aptamers in the therapeutics and diagnostics pipelines. Theranostics. 2018;8(15):4016-4032.

31. Hong P, Li W, Li J. Applications of aptasensors in clinical diagnostics. Sensors. 2012;12(2):1181-1193.

32. Pfeiffer F, Mayer G. Selection and biosensor application of aptamers for small molecules. Front Chem. 2016;4:25.

33. Liu Y, Tuleouva N, Ramanculov E, Revzin A. Aptamer-based electrochemical biosensor for interferon gamma detection. Anal Chem. 2010; 82(19):8131-8136.

34. Huang Y, Xu J, Liu J, Wang X, Chen B. Disease-related detection with electrochemical biosensors: a review. Sensors. 2017;17(10):2375.

35. Catanante G, Mishra RK, Hayat A, Marty JL. Sensitive analytical performance of folding based biosensor using methylene blue tagged aptamers. Talanta. 2016;153:138-144.

36. Dhiman A, Haldar S, Mishra SK, et al. Generation and application of DNA aptamers against HspX for accurate diagnosis of tuberculous meningitis. Tuberculosis. 2018;112:27-36.

37. Kalra P, Dhiman A, Cho WC, Bruno JG, Sharma TK. Simple Methods and Rational Design for Enhancing Aptamer Sensitivity and Specificity. Front Mol Biosci. 2018;14;5:41.

38. Das R, Sharma MK, Rao VK, et al. An electrochemical genosensor for Salmonella typhi on gold nanoparticles-mercaptosilane modified screen printed electrode. J Biotechnol. 2014;188:9-16.

39. Rufai SB, Singh A, Singh J, et al. Diagnostic usefulness of Xpert MTB/ RIF assay for detection of tuberculous meningitis using cerebrospinal fluid. J Infect. 2017;75(2):125-131.

40. Thakur R, Goyal R, Sarma S. Laboratory diagnosis of tuberculous meningitis - is there a scope for further improvement? J Lab Physicians. 2010;2(1):21-24.

41. Thwaites G, Chau TT, Mai NT, Drobniewski F, McAdam K, Farrar J. Tuberculous meningitis. J Neurol Neurosurg Psychiatry. 2000;68(3): 289-299.

42. Pai M, Flores LL, Pai N, Hubbard A, Riley LW, Colford JM. Diagnostic accuracy of nucleic acid amplification tests for tuberculous meningitis: a systematic review and meta-analysis. Lancet Infect Dis. 2003;3(10): 633-643.

43. Solomons RS, van Elsland SL, Visser DH, et al. Commercial nucleic acid amplification tests in tuberculous meningitis-a meta-analysis. Diagn Microbiol Infect Dis. 2014;78(4):398-403.

44. Haldar S, Sharma N, Gupta VK, Tyagi JS. Efficient diagnosis of tuberculous meningitis by detection of Mycobacterium tuberculosis DNA in cerebrospinal fluid filtrates using PCR. J Med Microbiol. 2009;58(5): 616-624.

45. Flores LL, Steingart KR, Dendukuri N, et al. Systematic review and meta-analysis of antigen detection tests for the diagnosis of tuberculosis. Clin Vaccine Immunol. 2011;18(10):1616-1627. 


\section{Supplementary materials Classification of cerebrospinal fluid (CSF) samples}

A subset of 81 pediatric CSF samples that was derived from an archived set of samples described previously by Haldar et $\mathrm{al}^{1}$ was used in the present study. The samples were categorized according to a universal case definition for tuberculous meningitis (TBM). ${ }^{2}$ Culture/smear/commercial nucleic acid amplification test (NAAT) positive/ acid-fast bacillus (AFB) seen on autopsy were categorized as "definite" TBM. "Probable" and "possible" TBM groups include subjects negative by the above criteria but satisfying the defined clinical criteria, CSF biochemistry and cytology, cerebral imaging criteria and evidence of extraneural TB having a score of $\geq 10-12$ ("probable" TBM) and a score of $\geq 6-11$ ("possible" TBM). In this CSF sample subset $(\mathrm{N}=81)$, samples were classified as "definite" TBM on the basis of $M$. tuberculosis culture positivity only $(\mathrm{N}=16)$ and as "probable" TBM ( $\mathrm{N}=7$, score range: 10-18), "possible" TBM ( $\mathrm{N}=16$, score range: 6-9) and "not-tuberculous meningitis" (not-TBM) with an alternative diagnosis established $(\mathrm{N}=42)$. The "not-TBM" category was further sub-classified into nontuberculous infectious meningitis (NTIM, N=16), infectious neurological disorders (IND, $\mathrm{N}=16$ ) and noninfectious neurological disorders (NIND, $\mathrm{N}=10$ ). The median age and range of the children in each diagnostic category was quite comparable (Table S1). The NTIM comprised of cases of pyogenic bacterial meningitis that included 2 culture-confirmed cases of E. coli $(\mathrm{N}=1)$, and Acinetobacter sp. $(\mathrm{N}=1)$. Another 14 cases were diagnosed on the basis of

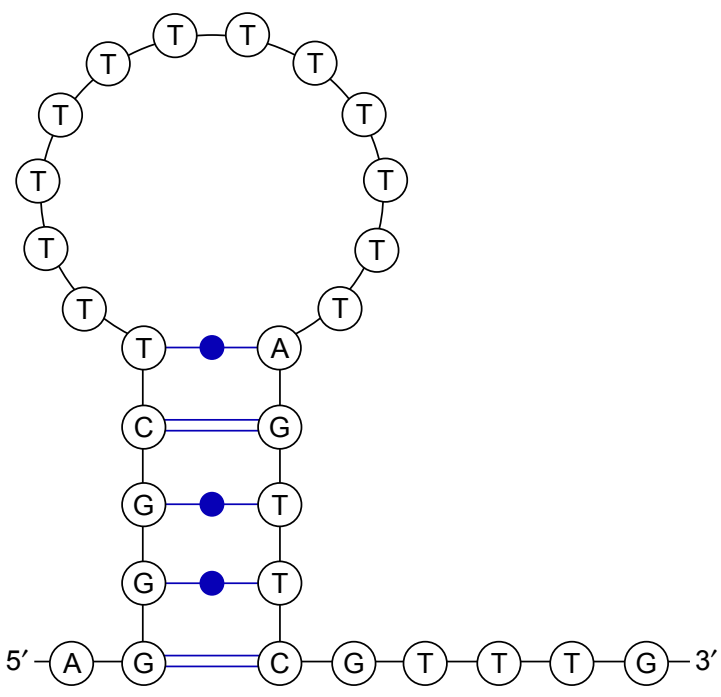

Figure SI Secondary structure of H63 SL-2 M6 aptamer.

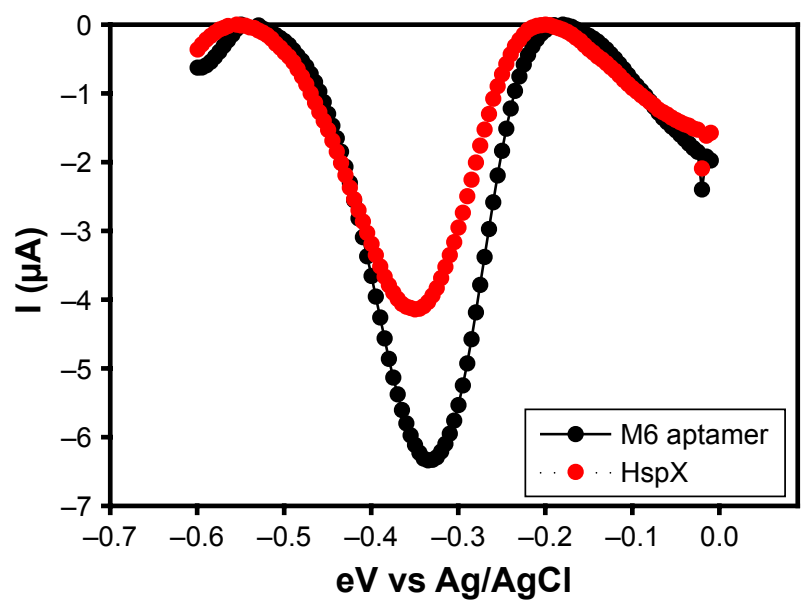

Figure S2 H63 SL-2 M6 Aptamer (black) and H63 SL-2 M6+HspX (red).

response to appropriate antibiotics, clinical presentation and symptoms. The IND category included 5 cases of meningoencephalitis, 4 cases of enteric encephalopathy, 3 cases of sepsis, 2 cases of cerebral malaria and 1 case each of pneumonia and post diphtheritic polyneuritis. The NIND category included 5 cases of neurodegenerative disorders, 2 cases each of hypocalcemic seizures and transverse myelitis, 1 case of Guillain-Barré syndrome.

\section{Circular dichroism (CD) study}

CD spectra were collected as described recently using Jasco J-815 Spectropolarimeter (Jasco Hachioji, Tokyo, Japan) equipped with Peltier junction temperature controller and continuous nitrogen flow was maintained to avoid water

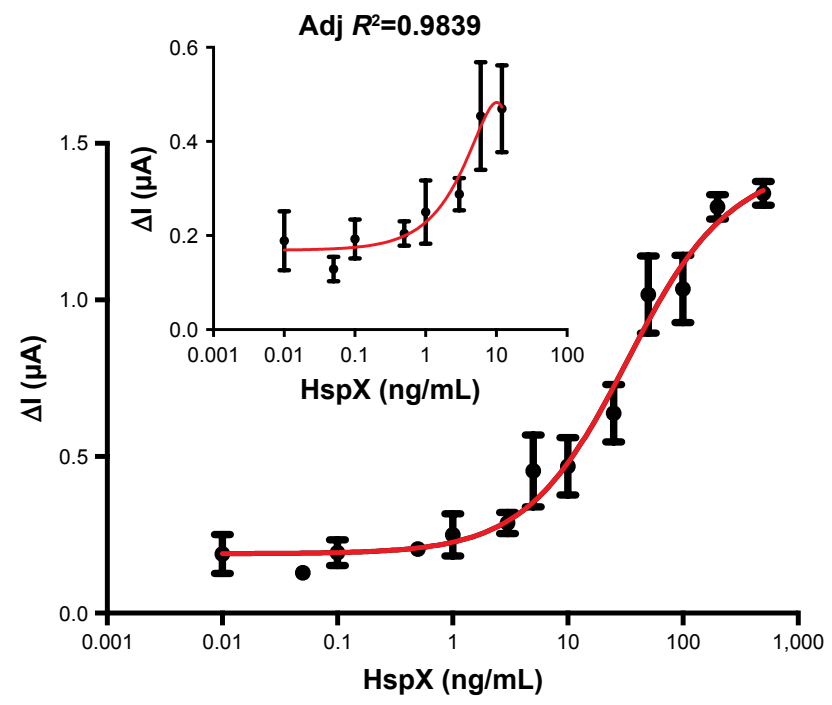

Figure S3 Sensor response as a function of protein ( $\mathrm{HspX})$ data was fitted using sigmoidal fitting equation in Origin Pro 8 software.

Note: Image inset depicts the limit of detection in the lower antigen range. 


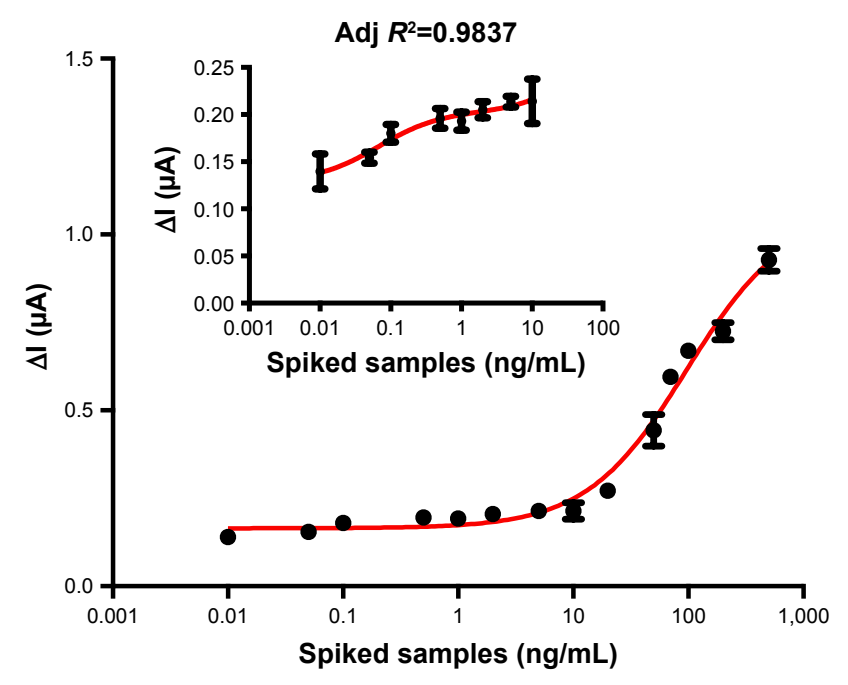

Figure S4 Sensor response as a function of protein (HspX) spiked in pooled CSF (NIND).

Notes: Samples and data were fitted using sigmoidal fitting equation in Origin Pro 8 software. Image inset depicts the limit of detection in the lower antigen range.

Abbreviations: CSF, cerebrospinal fluid; NIND, noninfectious neurological disorders. condensation around the cuvette as described recently. ${ }^{3}$ A cuvette with path length of $0.2 \mathrm{~cm}$ was used, each spectra was scanned at interval of $0.1 \mathrm{~nm}$ at scan rate of $20 \mathrm{~nm} / \mathrm{min}$ in binding buffer $(10 \mathrm{mM}$ Tris $\mathrm{pH} 7.5,10 \mathrm{mM} \mathrm{MgCl}, 50 \mathrm{mM}$ $\mathrm{KCl}, 25 \mathrm{mM} \mathrm{NaCl}$ ). Blank spectrum was recorded (binding buffer) and subtracted from the CD spectrum obtained from the aptamer or aptamer + HspX containing solution. Three scans were recorded for each sample in a range of 200-340 $\mathrm{nm}$ and their average was plotted to examine the target-dependent change in aptamer structure.

\section{Statistical analysis}

The diagnostic potential of the aptamer-based electrochemical sensor was evaluated by obtaining current difference (before and after adding the sample) values in CSF from "definite" TBM (true positives) and NTIM group (true negatives) as described recently. ${ }^{3}$ The obtained values were used

Table SI Comparison of various CSF parameters $(\mathrm{N}=8 \mathrm{I})^{\mathrm{a}}$

\begin{tabular}{|c|c|c|c|c|c|c|c|}
\hline Parameters & $\begin{array}{l}\text { TBM }^{\mathrm{b}} \\
(\mathrm{N}=39)\end{array}$ & $\begin{array}{l}\text { NTIM } \\
(\mathbf{N}=16)\end{array}$ & $\begin{array}{l}\text { IND } \\
(\mathbf{N}=16)\end{array}$ & $\begin{array}{l}\text { NIND } \\
(\mathbf{N}=10)\end{array}$ & $\begin{array}{l}\text { TBM } \\
\text { vs } \\
\text { NTIM }\end{array}$ & $\begin{array}{l}\text { TBM } \\
\text { vs } \\
\text { IND }\end{array}$ & $\begin{array}{l}\text { TBM } \\
\text { vs } \\
\text { NIND }\end{array}$ \\
\hline Age (years) & $7(2.75-11)$ & $6(1.31-10.35)$ & $7.5(4.5-13.25)$ & $5.5(1.75-10.5)$ & - & - & - \\
\hline Cells in CSF (per $\mu \mathrm{L}$ ) & $30(5.5-100)$ & $55(15-175)$ & $0.5(0-2.75)$ & $0(0-2)$ & $P=0.55$ & $\begin{array}{l}<0.05 \\
P=0.0003\end{array}$ & $<0.0001$ \\
\hline $\begin{array}{l}\text { Number of samples } \\
\text { with cells ( } \geq 10 \text { per } \mu \mathrm{L} \text { ) }\end{array}$ & 28 & 12 & 2 & 3 & - & - & - \\
\hline Lymphocyte \% & $75(24.5-100)$ & $5(0-65)$ & $25(0-100)$ & $0(0-100)$ & $\begin{array}{l}<0.05 \\
P=0.02\end{array}$ & $P=0.25$ & $P=0.22$ \\
\hline $\begin{array}{l}\text { Number of samples } \\
\text { with lymphocytes } \\
(>50 \%)\end{array}$ & 25 & 5 & 7 & 7 & - & - & - \\
\hline Neutrophils & $0(0-30)$ & $30(0-92.5)$ & $0(0-0)$ & $0(0-0)$ & $P=0.17$ & $P=0.29$ & $\begin{array}{l}<0.05 \\
P=0.003\end{array}$ \\
\hline CSF sugar (mg\%) & $53(34.5-66.5)$ & $54(25-79.75)$ & $69.5(60-77.5)$ & $62(48.75-82.25)$ & $P=0.83$ & $\begin{array}{l}<0.05 \\
P=0.013\end{array}$ & $P=0.14$ \\
\hline $\begin{array}{l}\text { Concomitant blood } \\
\text { sugar }\end{array}$ & $108(95.5-121.5)$ & $111(108-121)$ & $100(9|-| 16.5)$ & $99.5(92.75-110.25)$ & $P=0.34$ & $P=0.5 I$ & $P=0.4 I$ \\
\hline $\begin{array}{l}\text { CSF to blood sugar } \\
\text { ratio }\end{array}$ & $0.47(0.32-0.58)$ & $0.49(0.19-0.7)$ & $0.7(0.58-0.728)$ & $0.69(0.43-0.82)$ & $P=0.99$ & $\begin{array}{l}<0.05 \\
P=0.002\end{array}$ & $\begin{array}{l}<0.05 \\
P=0.02\end{array}$ \\
\hline $\begin{array}{l}\text { Number of samples } \\
\text { with low CSF to blood } \\
\text { sugar ratio }(<0.5)\end{array}$ & 19 & 8 & 3 & 5 & - & - & - \\
\hline CSF protein (mg\%) & $97(75-136)$ & $75(43.5-105.5)$ & $52(34.5-77.5)$ & $32.5(25.5-62.5)$ & $P=0.06$ & $\begin{array}{l}<0.05 \\
P=0.002\end{array}$ & $\begin{array}{l}<0.05 \\
P=0.0004\end{array}$ \\
\hline $\begin{array}{l}\text { Number of samples } \\
\text { with raised CSF } \\
\text { proteins }(>100 \mathrm{mg} / \mathrm{dL})\end{array}$ & 18 & 4 & 3 & 2 & - & - & - \\
\hline
\end{tabular}

Notes: alian values are indicated; values in brackets indicate the interquartile range. This study was performed on an archived sample from a previous study by Haldar et al.' 'Includes "definite", "probable" and "possible" TBM groups.

Abbreviations: TBM, tuberculosis meningitis; NTIM, nontuberculosis infectious meningitis; IND, infectious neurological disorders; NIND, nonIND; CSF, cerebrospinal fluid. 

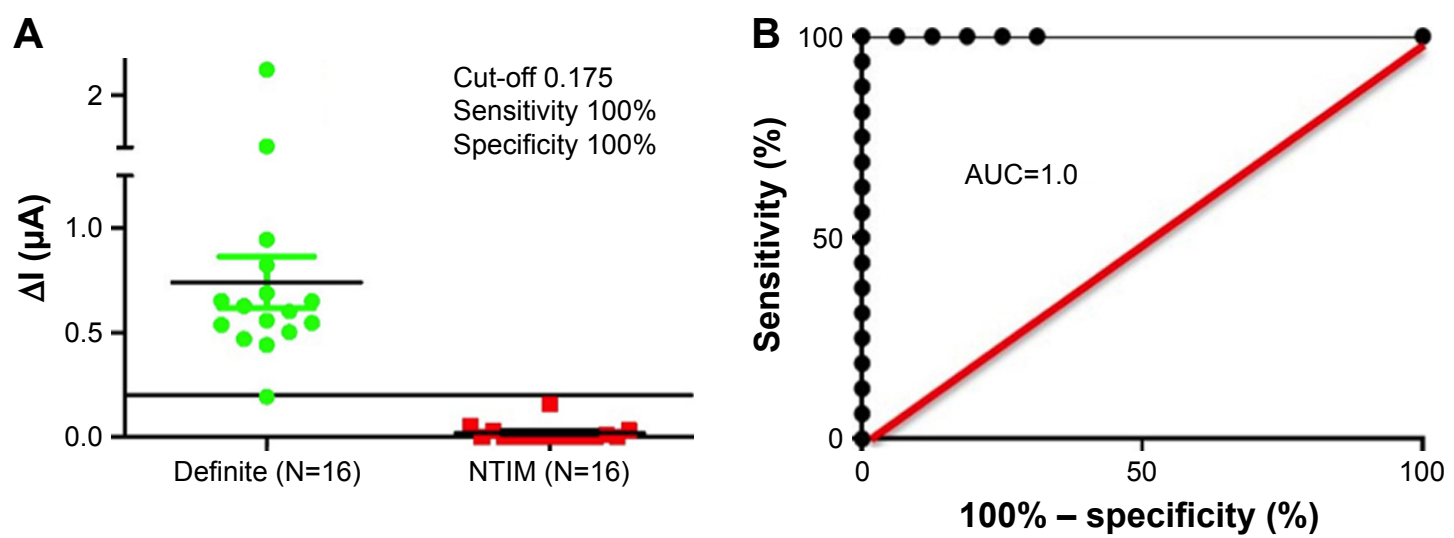

Figure S5 Scatter plot showing sensor response for CSF samples from definite (true positive) and NTIM (true negative) subjects (A). ROC curve derived from sensor response for definite (true positive) and NTIM (true negative) samples (B) showing AUC as 1.0 evincing robust performance of the sensor.

Abbreviations: NTIM, nontuberculous infectious meningitis; AUC, area under the curve; CSF, cerebrospinal fluid; ROC, receiver operating characteristic.

to plot receiver operating characteristic curve (ROC) using GraphPad Prism version 5.00 for Windows, (GraphPad Software, Inc., La Jolla, CA, USA, www.graphpad.com). The cut-off values were obtained to achieve test sensitivity ( $\geq 95 \%$ ) coherent with recent recommendations for diagnostic tests targeting childhood TB. These cut-off values were then applied to the "probable" and "possible" TBM groups, "IND" and "NIND" of not-TBM subjects to evaluate assay performance.

\section{References}

1. Haldar S, Sankhyan N, Sharma N, et al. Detection of Mycobacterium tuberculosis GlcB or HspX antigens or devR DNA impacts the rapid diagnosis of tuberculous meningitis in children. PLoS One. 2012;7(9): e44630.

2. Marais S, Thwaites G, Schoeman JF, et al. Tuberculous meningitis: a uniform case definition for use in clinical research. Lancet Infect Dis. 2010;10(11):803-812.

3. Dhiman A, Haldar S, Mishra SK, et al. Generation and application of DNA aptamers against HspX for accurate diagnosis of tuberculous meningitis. Tuberculosis. 2018;112:27-36.
International Journal of Nanomedicine

\section{Publish your work in this journal}

The International Journal of Nanomedicine is an international, peerreviewed journal focusing on the application of nanotechnology in diagnostics, therapeutics, and drug delivery systems throughou the biomedical field. This journal is indexed on PubMed Central, MedLine, CAS, SciSearch ${ }^{\circledR}$, Current Contents ${ }^{\circledR} /$ Clinical Medicine,

\section{Dovepress}

Journal Citation Reports/Science Edition, EMBase, Scopus and the Elsevier Bibliographic databases. The manuscript management system is completely online and includes a very quick and fair peer-review system, which is all easy to use. Visit http://www.dovepress.com/ testimonials.php to read real quotes from published authors. 